\title{
Reflets
}

Revue ontaroise d'intervention sociale et communautaire

\section{Le sacré : au coeur ou en marge du social?}

\section{Nérée St-Amand et Noël Simard}

Volume 12, numéro 1, 2006

Spiritualité et intervention sociale

URI : https://id.erudit.org/iderudit/013435ar

DOI : https://doi.org/10.7202/013435ar

Aller au sommaire du numéro

Éditeur(s)

Reflets : Revue ontaroise d'intervention sociale et communautaire

ISSN

1203-4576 (imprimé)

1712-8498 (numérique)

Découvrir la revue

Citer ce document

St-Amand, N. \& Simard, N. (2006). Le sacré : au coeur ou en marge du social? Reflets, 12(1), 10-15. https://doi.org/10.7202/013435ar

Tous droits réservés (C) Reflets : Revue ontaroise d'intervention sociale et communautaire, 2006
Ce document est protégé par la loi sur le droit d'auteur. L'utilisation des services d'Érudit (y compris la reproduction) est assujettie à sa politique d'utilisation que vous pouvez consulter en ligne.

https://apropos.erudit.org/fr/usagers/politique-dutilisation/ 


\section{Le sacré : au cœur ou en marge du social?}

\section{Nérée St-Amand et Noël Simard}

En ce début de $\mathrm{XXI}^{\mathrm{e}}$ siècle, plusieurs phénomènes sociaux se chevauchent et s'entrecroisent, tous aussi complexes les uns que les autres, dans une société où la mondialisation ajoute une dimension de plus à la complexité des analyses que nous tentons d'en faire. Le comité éditorial de Reflets s'est intéressé à deux facteurs en ébullition dans ce monde en mutation : d'une part, une remontée de la religion, voire d'un fondamentalisme religieux, et d'autre part, un intérêt accru pour la spiritualité, phénomène allant de pair, dans une certaine mesure, avec une remise en question des pratiques religieuses traditionnelles. Montée de la religiosité pour certains, distance face à une religion opprimante pour d'autres. Force est de constater que plusieurs groupes religieux prennent aujourd'hui plus de place, même sur le plan politique. Quelles sont, par exemple, les conséquences d'un rapprochement entre l'État et l'Église? D'un autre côté, un nombre de plus en plus important de personnes et de mouvements sociaux cherchent ailleurs que dans les religions traditionnelles une réponse aux questions existentielles, tant morales qu'éthiques ou sociales, qui se posent en sciences humaines et sociales, en particulier.

Nul doute que nous assistons à la fois à une montée de l'intérêt pour la spiritualité et à une renaissance du phénomène religieux. Il nous semble que ces deux mouvements soient possiblement convergents à l'occasion, parallèles en d'autres instances et parfois opposés dans le sens où une montée de la religiosité, surtout dans ses manifestations fondamentalistes, n'a peut-être rien à voir avec un intérêt accru pour la spiritualité. En fait, il peut s'agir 
de mouvements forts différents qui s'intéressent à des sujets plus ou moins conciliables et qui vont chercher des clientèles aux préoccupations divergentes.

Forts de ce double constat, nous avons opté pour une quête de sens, une réponse à quelques questions entourant la spiritualité en liens avec l'intervention sociale. C'est ainsi qu'en proposant ce numéro de Reflets, nous avions quelques objectifs en tête :

- Évaluer l'état de la recherche sur le sujet de la spiritualité, une spiritualité qui puisse s'appliquer aux professions d'aide; d'une part;

- Distinguer la spiritualité de la religion et tenter une percée dans le domaine des liens possibles entre spiritualité et intervention sociale;

- Déterminer les conséquences de tels mouvements sur l'intervention sociale;

- Proposer des pistes prometteuses qui puissent s'appliquer tout autant aux personnes travaillant dans les professions d'aide qu'aux organismes responsables des services humains et sociaux;

- Alimenter les débats sur la relation thérapeutique en y ajoutant la dimension spirituelle.

Un défi de taille, d'autant plus que la littérature sur le sujet de la spiritualité en intervention sociale est plutôt mince et que les chercheurs s'intéressant au sujet, soit en Ontario ou au Canada français, ne sont pas nécessairement légion. Qui plus est, sur le plan de l'enseignement, c'est le silence à peu près total dans ce domaine.

Deux ans après avoir accepté ce défi, nous sommes fiers de présenter un numéro original, qui pose des questions que peu de gens osent aborder, surtout en intervention sociale : quelle place occupe la spiritualité dans nos milieux, dans nos recherches, dans nos enseignements, dans nos interventions, mais aussi dans nos propres pratiques? En fait, quels sont les liens entre nos croyances et pratiques spirituelles et les interventions que nous posons? Quelles pratiques savons-nous reconnaitre ou encourager chez les gens qui dévoilent certaines croyances fondées sur une spiritualité plus ou moins définie? 
Aux auteurs et auteures qui nous ont proposé un article, nous avons demandé de définir les deux termes qui pourraient, au départ, porter à confusion : spiritualité et religion. Nous nous retrouvons donc avec plusieurs interprétations et définitions de l'une et de l'autre, basées sur la littérature consultée, les recherches en cours, les expériences et croyances personnelles. Nous leur avons aussi demandé de proposer des liens entre leurs recherches et l'intervention sociale. Vous pourrez donc lire dans Le Dossier six articles qui abordent plusieurs angles, qui décrivent plusieurs tendances, qui proposent plusieurs pistes.

En décembre 2005, nous voulions effectuer L'entrevue avec notre collègue et ami Brian Ouellet, de l'Université St-Thomas. Malheureusement, il est décédé subitement, au moment même où nous devions avoir l'entrevue en question. Nous lui dédions ce numéro. En même temps, nous avons décidé de ne pas recourir à une autre entrevue, symbolisant par là le vide qu'il laisse au Canada, dans tout le domaine de la spiritualité en intervention sociale.

\section{Le dossier}

Partant de sa formation et d'une expérience unique au Forum des cultures de Barcelone, Nérée St-Amand fait une percée dans le monde largement inexploré de la spiritualité dans l'intervention sociale et explore quelques pistes de réflexion en découlant. Bien conscient du fait que la spiritualité évolue de plusieurs façons et selon divers principes, il conclut qu'il est urgent de changer de cap; c'est en ce sens qu'il propose un service social à caractère spirituel. Il suggère alors certains principes qui pourraient guider la pratique de l'intervention sociale dans une perspective holistique, au cœur de laquelle se retrouve la dimension spirituelle. Par ricochet, le choix de ces principes remet en question des pratiques qui justement négligent ou même évacuent la dimension sacrée. En fait, l'auteur propose carrément un travail social basé sur la spiritualité. Les pistes et exemples qu'il décrit sont présentés comme autant de 
chemins qui peuvent faciliter l'intégration de la spiritualité en intervention sociale.

Louis-Charles Lavoie analyse les liens entre la psychothérapie et la spiritualité et montre comment on passe actuellement d'une attitude d'opposition à un dialogue interactif entre les deux. Après avoir présenté les auteurs et les courants principaux qui se sont opposés à toute ingérence spirituelle dans le champ de la psychothérapie ou qui ont réintroduit les notions d'âme et de spiritualité dans ce domaine, l'auteur analyse les changements qui ont relancé la question de la place de la spiritualité en psychothérapie et décrit l'évolution de cette question depuis les dernières décennies, en se concentrant en particulier sur deux représentants de l'école humaniste. Même si cette approche du dialogue et de l'interaction soulève encore résistances, doutes et oppositions, elle suscite un intérêt grandissant, tant dans la littérature que dans les milieux de pratique.

Pour sa part, Mohammed Khalid s'est penché sur les liens entre le service social, la spiritualité et le vieillissement. Dans un premier temps, il examine les trois composantes de la problématique à l'étude, à savoir les origines religieuses et spirituelles du service social, le statut académique de la gérontologie, et le concept polysémique de la spiritualité. L'auteur résume ensuite la théorie géronto-transcendantale et analyse les raisons invoquées par les partisans et les adversaires de l'inclusion de la gérontologie et de la spiritualité dans la formation des travailleurs et travailleuses sociales. Enfin, et c'est là un autre apport original de son article, il propose un modèle conceptuel qui pourra faire avancer le débat et contribuer à l'émergence d'attitudes favorables tant à la gérontologie qu'à la spiritualité. Khalid démontre que les sphères du service social, de la gérontologie et de la spiritualité forment une suprasphère au sein de laquelle il est possible de parler d'un service social géronto-spirituel ou géronto-transcendantal.

Noël Simard pour sa part s'intéresse à la notion de la spiritualité et à l'influence de cette dernière sur la santé.Après avoir proposé un modèle pour bien décrire la spiritualité, l'auteur situe d'une manière critique la centralité de la réflexion sur la spiritualité dans les soins de santé. Il présente certains facteurs pouvant expliquer 
l'intérêt croissant pour la spiritualité dans le monde de la santé ainsi qu'un bilan des recherches sur son influence et sur la place qu'elle y tient. Reconnaitre la dimension spirituelle de la santé découle d'une vision globale de la médecine. Cependant, l'intégration de la dimension spirituelle dans les soins et les services de santé requiert une clarification du concept de même qu'une prise en compte à la fois de la distinction et de la complémentarité des champs spirituel et thérapeutique.

Dans l'article Vivre intensément l'humain : une exploration du deuil après le VIH-sida, les auteurs Cadell et Haubrich explorent l'expérience de quinze individus ayant pris soin de personnes décédées é la suite des complications dues au VIH-sida. Après avoir présenté le cadre de leur recherche, les auteurs démontrent en quel sens la spiritualité est habituellement perçue comme une expérience positive, diversifiée, enrichissante. Se concentrant sur l'engagement face au sida, à la mort, au monde, à la personne décédée, les auteurs concluent que la spiritualité constitue un facteur important d'adaptation permettant aux soignants de donner ou de redonner sens à leur vie é la suite d'expériences particulièrement difficiles.

Le dernier article, et non le moindre, est le fruit d'une longue recherche entreprise par Pierre Ouellette et Dom Raymond Carette; il porte sur les motivations à faire une retraite individuelle dans un monastère bénédictin. Ce texte nous interpelle sur le plan de la quête de sens des gens qui fréquentent un tel établissement. Après avoir présenté les grands traits de la spiritualité bénédictine et la théorie de la restauration de l'attention dirigée, les auteurs démontrent comment le monastère de Saint-Benoît-du-Lac semble satisfaire les exigences d'un environnement fortifiant dont les quatre propriétés principales sont d'être un lieu de retrait, d'environnement d'ampleur, de fascination et de comptabilité (entendue comme correspondance entre les intentions et les objectifs des retraitants et les caractéristiques de l'environnement). Par la suite, le texte regroupe les différentes motivations selon les facteurs de la spiritualité, de la beauté, de la réflexion et de la réceptivité. Loin de se limiter à une étude quantitative, le texte propose, toujours dans un souci de recherche de sens, plusieurs 
réflexions des gens ayant participé à cette étude. Enfin, les auteurs dégagent quelques implications pour l'intervention sociale, telle la reconnaissance des bienfaits de la prière, du silence et de la méditation.

\section{Des pratiques à notre image}

Cinq auteurs ont abordé le sujet de la spiritualité sous un angle plus personnel, moins théorique. Présentés sous forme de texte d'opinion, de partage d'expériences ou de témoignages, leurs articles confirment la nécessité de sortir la spiritualité du placard, tant sur le plan de la pratique de l'intervention sociale que sur celui de la formation à l'intervention. Ces auteurs et auteures abordent à la fois les défis à relever pour intégrer la dimension spirituelle dans la formation et l'engagement personnel nécessaire pour faire en sorte que les pratiques collent au vécu des intervenantes et intervenants. Cette intégration nous rappelle que la réponse à nos besoins fondamentaux doit venir non pas tant d'une quête de science que d'une quête de sens. Sur ce plan, la spiritualité joue un rôle crucial, un rôle sacré.

Il nous semble que l'ensemble du dossier envoie un message clair à savoir que des pratiques d'intervention spirituelles existent; elles sont multiformes, vivantes, mais souvent silencieuses; en fait, elles semblent devancer les recherches et l'enseignement. Si tel est le cas, les intellectuels et les professionnels de l'aide se doivent de mettre la spiritualité à l'agenda des recherches, des enseignements, de la formation, pour que les arrimages possibles entre diverses visions de l'intervention puissent susciter les débats qui s'imposent. 